\title{
MACROFUNGI DETERMINED IN UZUNGÖL NATURE PARK (TRABZON)
}

\author{
Ilgaz AKATA ${ }^{1 *}$, Yasin $\mathrm{UZUN}^{2}$ \\ ${ }^{1}$ Ankara University, Faculty of Science, Department of Biology, Ankara, Turkey \\ ${ }^{2}$ Karamanoğlu Mehmetbey University, Kamil Özdağ Science Faculty, Department of Biology, Karaman, Turkey \\ *Corresponding author: akata@science.ankara.edu.tr
}

Received (Alınış): 28 Fabruary 2017, Accepted (Kabul): 20 March 2017, Online First (Erken Görünüm): 4 April 2017, Published (Basım): 15 June 2017

\begin{abstract}
In the present study, macrofungi samples collected from Uzungöl Nature Park (Trabzon) between 2011 and 2013 were identified and classified. After field and laboratory studies, a total of 205 macrofungi species were determined. A list of 212 species, by including the previously reported 7 species in the research area, belonging to 129 genera and 64 families within 2 divisions were given. Fourty-six species were determined to belong to Ascomycota and 166 to Basidiomycota.
\end{abstract}

Key words: Biodiversity, macrofungi, Uzungöl Nature Park, Turkey.

\section{Uzungöl Tabiat Parkı (Trabzon)'ndan Belirlenen Makrofunguslar}

Özet: Bu çalışmada, Uzungöl Tabiat Parkı (Trabzon)'ndan 2011 ve 2013 yılları arasında toplanan makrofungus örnekleri teşhis edilmiş ve sınıflandırılmıştır. Arazi ve laboratuvar çalışmaları sonrasında 205 makromantar türü tespit edilmiştir. Daha önceden araştırma alanından rapor edilmiş 7 tür de dâhil olmak üzere, toplam 2 bölüm içinde yer alan 64 familya ve 129 cinse ait toplam 212 tür verilmiştir. Tespit edilen türlerden 46's1 Ascomycota, 166's1 Basidiomycota bölümüne mensuptur.

Anahtar kelimeler: Biyoçeşitlilik, makromantarlar, Uzungöl Tabiat Parkı, Türkiye.

\section{Introduction}

The current available data on macrofungal diversity in Turkey is based on studies dating back to more than 100 years ago. Studies concerning macrofungi showed a considerable increase in number over the last decade and a genaral overview of the published data was given in Sesli and Denchev (2008). More recently, new studies on macrofungal diversity of Turkey yielded additional data (Akata et al. 2016a,b, All1 et al. 2016, Demirel et al. 2016, Doğan \& Kurt 2016, Doğan \& Öztürk 2015, Dülger \& Akata 2016, Sesli et al. 2016). Although a number of mycological investigations in and around the current study area (Uzungöl Nature Park) were performed (Akata 2012a,b, Akata \& Doğan 2015, Akata \& Kaya 2012, 2013a,b, Akata et al. 2011, 2012, 2014, 2016a,b, Baydar \& Sesli 1994, Kaya et al. 2013, Sesli 1993, Sesli et al. 2016 and Uzun et al. 2014, 2015), no detailed taxonomic investigation on macrofungal diversity here was carried out.

Uzungöl is a mountain lake situated in Eastern Black Sea region of Turkey at an elevation of approximately $1000 \mathrm{~m}$ asl. The lake is $105 \mathrm{~km}$ far to Trabzon Province and $25 \mathrm{~km}$ to Çaykara district center. The part of the lake area covering 1625 hectares was announced as Nature Park in 1989 considering of its rich biodiversity, wild life and beautiful landscape (Figure 1).

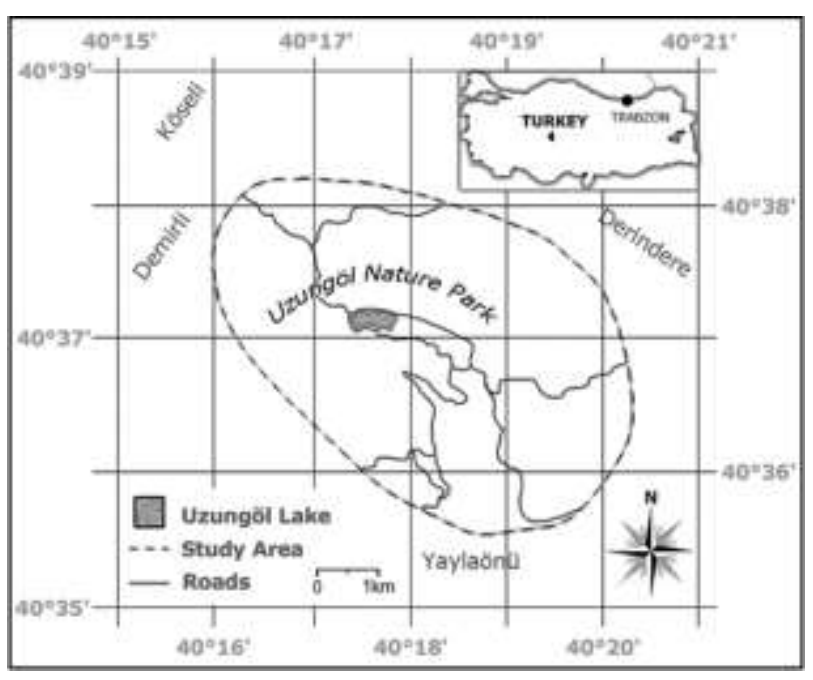

Figure 1. Map showing the study area around Uzungöl Nature Park. The borders of the sampled area was shown with dashed lines.

The forest vegetation of the Nature Park is primarily dominated by spruce (Picea orientalis L.), sometimes forming mixed stands with beech (Fagus orientalis Lipsky), fir (Abies nordmanniana (Stev.) Spach), alder (Alnus glutinosa L.), Scots pine (Pinus sylvestris L.), and several bushes such as hazelnut (Corylus avellana L.), 
common box (Buxus sempervirens L.) and common rhododendron (Rhododendron ponticum L.) are also present (Arikan et al. 2007, Atasoy 2010).

The region where the study lake is located has a typical oceanic climate with plentiful precipitation. The annual average temperature is $8^{\circ} \mathrm{C}$ and the rainfall is $926.4 \mathrm{~mm}$. Winters are snowy and cold with a lowest average temperature value of $0.1^{\circ} \mathrm{C}$, in February. Summers are warm, humid and the average maximum temperature is $15.38^{\circ} \mathrm{C}$, in July (Verep et al. 2002).

The purpose of the current study is to determine the macrofungal diversity of Uzungöl Nature Park and to make a contribution to the mycobiota of Turkey.

\section{Materials and Methods}

The fungal samples were collected between 2011 and 2013 from 40 localities inside the boundaries of Uzungöl Nature Park (Table 1). Ecological and morphological characteristics of the samples were recorded in the field and all samples were photographed in their natural environment. Samples were brought to herbarium for their general macroscopic and microscopic examinations. Identifications of the samples were performed according to Bas et al. (1988, 1990, 1995), Boertmann (1996), Breitenbach \& Kränzlin (1984, 1986, 1991, 1995, 2000), Fan et al. (1994), Hansen \& Knudsen (1992, 1997, 2000), Kränzlin (2005), Kuyper (1986), Medardi (2006), Pegler et al. (1997), Ryvarden \& Gilbertson (1993) and Sunhede (1989). The identified samples are deposited in Ankara University Herbarium (ANK).

\section{Results}

The identifications of the collected samples revealed presence of 205 macrofungal taxa in the studied area. The list of the identified species were given below with their locality, habitat, altitude, geographical coordinate, collection date and accesion number details. Names of all identified taxa were given in the list according to Cannon \& Kirk (2007) and Kirk et al. (2008).

Table 1. Coordinates, altitudes and positions of the sampled localities with respect to the lake.

\begin{tabular}{|c|c|c|c|}
\hline Locality No & $\begin{array}{l}\text { Locations of the sampling localities with respect } \\
\text { to the lake as the reference point }\end{array}$ & Coordinates & Altitudes (m) \\
\hline 1 & North-west of the Lake & $40^{\circ} 38^{\prime} 10^{\prime \prime} \mathrm{N}-40^{\circ} 16^{\prime} 17^{\prime \prime} \mathrm{E}$ & 920 \\
\hline 2 & North-west of the Lake & $40^{\circ} 37^{\prime} 26^{\prime \prime} \mathrm{N}-40^{\circ} 16^{\prime} 57^{\prime \prime} \mathrm{E}$ & 1100 \\
\hline 3 & North-west of the Lake & $40^{\circ} 37^{\prime} 08^{\prime \prime} \mathrm{N}-40^{\circ} 16^{\prime} 17^{\prime \prime} \mathrm{E}$ & 1600 \\
\hline 4 & South-west of the Lake & $40^{\circ} 36^{\prime} 59^{\prime \prime} \mathrm{N}-40^{\circ} 17^{\prime} 11^{\prime \prime} \mathrm{E}$ & 1300 \\
\hline 5 & South of the Lake & $40^{\circ} 36^{\prime} 44^{\prime \prime} \mathrm{N}-40^{\circ} 17^{\prime} 49^{\prime \prime} \mathrm{E}$ & 1540 \\
\hline 6 & South-east of the Lake & $40^{\circ} 36^{\prime} 30^{\prime \prime} \mathrm{N}-40^{\circ} 18^{\prime} 20^{\prime \prime} \mathrm{E}$ & 1580 \\
\hline 7 & South-east of the Lake & $40^{\circ} 36^{\prime} 23^{\prime \prime} \mathrm{N}-40^{\circ} 18^{\prime} 07^{\prime \prime} \mathrm{E}$ & 1835 \\
\hline 8 & South-east of the Lake & $40^{\circ} 36^{\prime} 15^{\prime \prime} \mathrm{N}-40^{\circ} 18^{\prime} 31^{\prime \prime} \mathrm{E}$ & 1470 \\
\hline 9 & South-east of the Lake & $40^{\circ} 35^{\prime} 56^{\prime \prime} \mathrm{N}-40^{\circ} 18^{\prime} 27^{\prime \prime} \mathrm{E}$ & 1690 \\
\hline 10 & South-east of the Lake & $40^{\circ} 35^{\prime} 38^{\prime \prime} \mathrm{N}-40^{\circ} 18^{\prime} 26^{\prime \prime} \mathrm{E}$ & 1710 \\
\hline 11 & North-east of the Lake & $40^{\circ} 37^{\prime} 52^{\prime \prime} \mathrm{N}-40^{\circ} 18^{\prime} 24^{\prime \prime} \mathrm{E}$ & 1620 \\
\hline 12 & North of the Lake & $40^{\circ} 37^{\prime} 50^{\prime \prime} \mathrm{N}-40^{\circ} 17^{\prime} 30^{\prime \prime} \mathrm{E}$ & 1450 \\
\hline 13 & North of the Lake & $40^{\circ} 37^{\prime} 21^{\prime \prime} \mathrm{N}-40^{\circ} 17^{\prime} 33^{\prime \prime} \mathrm{E}$ & 1290 \\
\hline 14 & North-east of the Lake & $40^{\circ} 37^{\prime} 24^{\prime \prime} \mathrm{N}-40^{\circ} 18^{\prime} 04^{\prime \prime} \mathrm{E}$ & 1400 \\
\hline 15 & North-east of the Lake & $40^{\circ} 37^{\prime} 17^{\prime \prime} \mathrm{N}-40^{\circ} 18^{\prime} 31^{\prime \prime} \mathrm{E}$ & 1360 \\
\hline 16 & North of the Lake & $40^{\circ} 38^{\prime} 10^{\prime \prime} \mathrm{N}-40^{\circ} 17^{\prime} 13^{\prime \prime} \mathrm{E}$ & 1350 \\
\hline 17 & North of the Lake & $40^{\circ} 37^{\prime} 38^{\prime \prime} \mathrm{N}-40^{\circ} 18^{\prime} 45^{\prime \prime} \mathrm{E}$ & 1800 \\
\hline 18 & North-west of the Lake & $40^{\circ} 38^{\prime} 28^{\prime \prime} \mathrm{N}-40^{\circ} 16^{\prime} 33^{\prime \prime} \mathrm{E}$ & 1230 \\
\hline 19 & West of the Lake & $40^{\circ} 38^{\prime} 35^{\prime \prime} \mathrm{N}-40^{\circ} 16^{\prime} 12^{\prime \prime} \mathrm{E}$ & 890 \\
\hline 20 & North-west of the Lake & $40^{\circ} 37^{\prime} 54^{\prime \prime} \mathrm{N}-40^{\circ} 16^{\prime} 12^{\prime \prime} \mathrm{E}$ & 1005 \\
\hline 21 & West of the Lake & $40^{\circ} 37^{\prime} 06^{\prime \prime} \mathrm{N}-40^{\circ} 16^{\prime} 26^{\prime \prime} \mathrm{E}$ & 1490 \\
\hline 22 & South of the Lake & $40^{\circ} 36^{\prime} 29^{\prime \prime} \mathrm{N}-40^{\circ} 17^{\prime} 34^{\prime \prime} \mathrm{E}$ & 1500 \\
\hline 23 & North-east of the Lake & $40^{\circ} 37^{\prime} 11^{\prime \prime} \mathrm{N}-40^{\circ} 18^{\prime} 24^{\prime \prime} \mathrm{E}$ & 1200 \\
\hline 24 & East of the Lake & $40^{\circ} 37^{\prime} 00^{\prime \prime} \mathrm{N}-40^{\circ} 18^{\prime} 41^{\prime \prime} \mathrm{E}$ & 1160 \\
\hline 25 & South of the Lake & $40^{\circ} 36^{\prime} 52^{\prime \prime} \mathrm{N}-40^{\circ} 17^{\prime} 36^{\prime \prime} \mathrm{E}$ & 1260 \\
\hline 26 & East of the Lake & $40^{\circ} 36^{\prime} 57^{\prime \prime} \mathrm{N}-40^{\circ} 18^{\prime} 02^{\prime \prime} \mathrm{E}$ & 1290 \\
\hline 27 & East of the Lake & $40^{\circ} 36^{\prime} 35^{\prime \prime} \mathrm{N}-40^{\circ} 18^{\prime} 31^{\prime \prime} \mathrm{E}$ & 1440 \\
\hline 28 & East of the Lake & $40^{\circ} 36^{\prime} 36^{\prime \prime} \mathrm{N}-40^{\circ} 18^{\prime} 04^{\prime \prime} \mathrm{E}$ & 1700 \\
\hline 29 & North-east of the Lake & $40^{\circ} 37^{\prime} 10^{\prime \prime} \mathrm{N}-40^{\circ} 18^{\prime} 44^{\prime \prime} \mathrm{E}$ & 1340 \\
\hline 30 & North-east of the Lake & $40^{\circ} 37^{\prime} 13^{\prime \prime} \mathrm{N}-40^{\circ} 18^{\prime} 53^{\prime \prime} \mathrm{E}$ & 1400 \\
\hline 31 & East of the Lake & $40^{\circ} 36^{\prime} 56^{\prime \prime} \mathrm{N}-40^{\circ} 18^{\prime} 52^{\prime \prime} \mathrm{E}$ & 1290 \\
\hline 32 & North-west of the Lake & $40^{\circ} 37^{\prime} 06^{\prime \prime} \mathrm{N}-40^{\circ} 16^{\prime} 43^{\prime \prime} \mathrm{E}$ & 1295 \\
\hline 33 & North-west of the Lake & $40^{\circ} 36^{\prime} 55^{\prime \prime} \mathrm{N}-40^{\circ} 16^{\prime} 32^{\prime \prime} \mathrm{E}$ & 1500 \\
\hline 34 & North-west of the Lake & $40^{\circ} 37^{\prime} 00^{\prime \prime} \mathrm{N}-40^{\circ} 16^{\prime} 28^{\prime \prime} \mathrm{E}$ & 1540 \\
\hline 35 & West of the Lake & $40^{\circ} 36^{\prime} 56^{\prime \prime} \mathrm{N}-40^{\circ} 16^{\prime} 46^{\prime \prime} \mathrm{E}$ & 1380 \\
\hline 36 & West of the Lake & $40^{\circ} 36^{\prime} 49^{\prime \prime} \mathrm{N}-40^{\circ} 16^{\prime} 34^{\prime \prime} \mathrm{E}$ & 1370 \\
\hline 37 & North of the Lake & $40^{\circ} 38^{\prime} 15^{\prime \prime} \mathrm{N}-40^{\circ} 17^{\prime} 46^{\prime \prime} \mathrm{E}$ & 1560 \\
\hline 38 & North of the Lake & $40^{\circ} 37^{\prime} 38^{\prime \prime} \mathrm{N}-40^{\circ} 17^{\prime} 54^{\prime \prime} \mathrm{E}$ & 1650 \\
\hline 39 & North of the Lake & $40^{\circ} 37^{\prime} 14^{\prime \prime} \mathrm{N}-40^{\circ} 17^{\prime} 50^{\prime \prime} \mathrm{E}$ & 1180 \\
\hline 40 & East of the Lake & $40^{\circ} 36^{\prime} 45^{\prime \prime} \mathrm{K}-40^{\circ} 19^{\prime} 07^{\prime \prime} \mathrm{E}$ & 1430 \\
\hline
\end{tabular}




\section{Pylum AsCOMYCOTA}

Family Caloscyphaceae

Caloscypha fulgens (Pers.) Boud.: Locality 7, spruce and beech mixed forest, 16.05.2013, Akata \& Yuzun 1670 .

\section{Family Chlorociboriaceae}

Chlorociboria aeruginosa (Oeder) Seaver ex C.S. Ramamurthi, Korf \& L.R. Batra: Locality 14, on beech branch, 01.10.2011, Akata \& Yuzun 953.

\section{Family Cudoniaceae}

Cudonia circinans (Pers.) Fr.: Locality 17, spruce forest, 06.09.2011, Akata \& Yuzun 889.

Spathularia flavida Pers.: Locality 10, spruce forest, 25.09.2011, Akata \& Yuzun 947.

Spathulariopsis velutipes (Cooke \& Farl. ex Cooke) Maas Geest. (Akata \& Kaya 2013a).

\section{Family Diatrypaceae}

Diatrype disciformis (Hoffm.) Fr.: Locality 35, on beech branch, 17.10.2012, Akata \& Yuzun 1406.

\section{Family Geoglossaceae}

Trichoglossum hirsutum (Pers.) Boud. (Akata \& Kaya 2013a).

\section{Family Helotiaceae}

Ascocoryne cylichnium (Tul.) Korf.: Locality 28, 19.09.2012, on beech stump, Akata \& Yuzun 1202.

Bisporella citrina (Batsch) Korf \& S.E. Carp.: Locality 5, 10.09.2011, on beech stump, Akata \& Yuzun 910.

Cyathicula coronata (Bull.) Rehm (Akata 2012a).

Cyathicula cyathoidea (Bull.) Thüm.: Locality 1, on blackberry stem, 23.08.2011, Akata \& Yuzun 869.

Hymenoscyphus calyculus (Sowerby) W. Phillips: Locality 18, on beech branch, 11.09.2011, Akata \& Yuzun 923.

\section{Family Helvellaceae}

Helvella atra J. König: Locality 19, near spruce and beech mixed forest, 03.10.2011, Akata \& Yuzun 973.

Helvella crispa (Scop.) Fr.: Locality 9, spruce and beech mixed forest, 15.09.2011, Akata \& Yuzun 935.

Helvella elastica Bull.: Locality 10, near spruce forest, 25.09.2011, Akata \& Yuzun 952.

Helvella lactea Boud.: Locality 25, near spruce and beech mixed forest, 14.10.2011, Akata \& Yuzun 1023.

\section{Family Hyaloscyphaceae}

Neodasyscypha cerina (Pers.) Spooner: Locality 36, on beech branch, 26.10.2012, Akata \& Yuzun 1473.

\section{Family Hypocreaceae}

Hypocrea leucopus (P. Karst.) H.L. Chamb.: (Akata 2012b).

\section{Incertae sedis}

Tapesia fusca (Pers.) Fuckel: Locality 40, on common hazel branch, 27.10.2012, Akata \& Yuzun 1610.

\section{Family Lachnaceae}

Lachnum virgineum (Batsch) P. Karst.: Locality 4, on herbaceous stem, 06.09.2011, Akata \& Yuzun 888.

\section{Family Leotiaceae}

Leotia lubrica (Scop.) Pers.: Locality 14, spruce and beech mixed forest, 01.10.2011, Akata \& Yuzun 960.

\section{Family Morchellaceae}

Morchella esculenta (L.) Pers.: Locality 33, near spruce forest, 15.05.2012, Akata \& Yuzun 1042.

\section{Family Pezizaceae}

Peziza badia Pers.: Locality 5, spruce and beech mixed forest, 10.09.2011, Akata \& Yuzun 903.

Peziza cerea Sowerby: Locality 35, spruce and beech mixed forest, 17.10.2012, Akata \& Yuzun 1395.

Peziza saniosa Schrad.: (Akata \& Kaya 2012).

\section{Family Pyronemataceae}

Aleuria aurantia (Pers.) Fuckel: Locality 1, near road, 23.08.2011, Akata \& Yuzun 871.

Cheilymenia granulata (Bull.) J. Moravec: Locality 24, pastureland, on cow dung, 13.10.2011, Akata \& Yuzun 1014.

Humaria hemisphaerica (F.H. Wigg.) Fuckel: Locality 26, 08.09.2012, Akata \& Yuzun 1147.

Otidea alutacea (Pers.) Massee: Locality 10, spruce and beech mixed forest, 25.09.2011, Akata \& Yuzun 948.

Otidea onotica (Pers.) Fuckel: Locality 15, spruce forest 02.11.2011, Akata \& Yuzun 969.

Neottiella rutilans (Fr.) Dennis (Akata \& Kaya 2013b).

Rhodoscypha ovilla (Peck) Dissing \& Sivertsen (Akata \& Kaya 2013b).

Scutellinia scutellata (L.) Lambotte: Locality 5, spruce and beech mixed forest, 10.09.2011, Akata \& Yuzun 905.

Tarzetta catinus (Holmsk.) Korf \& J.K. Rogers: Locality 18, spruce and beech mixed forest, 11.09.2011, Akata \& Yuzun 918.

Tarzetta cupularis (L.) Svrcek: Locality 30, spruce and beech mixed forest, 19.10.2011, Akata \& Yuzun 1031.

\section{Family Rutstroemiaceae}

Lanzia echinophila (Bull.) Korf: Locality 1, under chestnut, inner surface of chestnut husk, 15.09.2011, Akata \& Yuzun 944.

\section{Family Sarcoscyphaceae}

Sarcoscypha coccinea (Jacq.) Sacc.: Locality 26, on beech branch, 08.09.2012, Akata \& Yuzun 1121. 


\section{Family Sarcosomataceae}

Plectania rhytidia (Berk.) Nannf. \& Korf: Locality 40, on common hazel branch, 27.10.2012, Akata \& Yuzun 1534 .

\section{Family Xylariaceae}

Daldinia concentrica (Bolton) Ces. \& De Not.: Locality 3, on beech branch, 23.08.2011, Akata \& Yuzun 875.

Hypoxylon fragiforme (Pers.: Fr.) J. Kickx f.: Locality 38, on beech branch, 26.10.2012, Akata \& Yuzun 1467.

Hypoxylon multiforme (Fr.) Fr.: Locality 4, on alder branch, 06.09.2011, Akata \& Yuzun 897.

Kretzschmaria deusta (Hoffm.) P.M.D. Martin: Locality 31, on beech stump, 20.10.2011, Akata \& Yuzun 1037.

Xylaria carpophila (Pers.) Fr.: Locality 8, on beech husk, 11.09.2011, Akata \& Yuzun 931.

Xylaria hypoxylon (L.) Grev.: Locality 36, on beech branch, 26.10.2012, Akata \& Yuzun 1501.

Xylaria longipes Nitschke: Locality 18, on beech stump, 11.09.2011, Akata \& Yuzun 919.

Xylaria polymorpha (Pers.) Grev.: Locality 25, on beech stump, 14.10.2011, Akata \& Yuzun 1021.

\section{Phylum BASIDIOMYCOTA}

\section{Family Agaricaceae}

Agaricus augustus Fr.: Locality 13, spruce forest, 01.10.2011, Akata \& Yuzun 952.

Agaricus luteomaculatus (F.H. Møller) F.H. Møller: Locality 37, spruce forest, 26.10.2012, Akata \& Yuzun 1470 .

Agaricus xanthodermus Genev.: Locality 8, spruce and beech mixed forest, 11.09.2011, Akata \& Yuzun 928.

Bovista nigrescens Pers.: Locality 40, spruce and beech mixed forest,27.10.2012, Akata \& Yuzun 1524.

Bovista plumbea Pers.: Locality 5, 06.09.2011, spruce and beech mixed forest, on path, Akata \& Yuzun 900.

Chlorophyllum rhacodes (Vittad.) Vellinga: Locality 25, spruce and beech mixed forest, 14.10.2011, Akata \& Yuzun 1027.

Coprinus comatus (O.F. Müll.) Pers.: Locality 2, pastureland, 23.08.2011, Akata \& Yuzun 874.

Crucibulum laeve (Huds.) Kambly: Locality 27, on beech branch, 08.09.2012, Akata \& Yuzun 1134.

Cyathus olla (Batsch) Pers.: Locality 39, 27.10.2012, on beech branch, Akata \& Yuzun 1509.

Cyathus striatus (Huds.) Willd.: Locality 29, on beech branch, 19.09.2012, Akata \& Yuzun 1166.
Cystodermella granulosa (Batsch) Harmaja: Locality 36, spruce and beech mixed forest, 26.10.2012, Akata \& Yuzun 1479.

Lepiota clypeolaria (Bull.) P. Kumm.: Locality 18, spruce and beech mixed forest, 11.09.2011, Akata \& Yuzun 920.

Lepiota cristata (Bolton) P. Kumm.: Locality 21, near road, 10.10.2011, Akata \& Yuzun 987.

Lepiota magnispora Murrill: Locality 39, spruce and beech mixed forest, 27.10.2012, Akata \& Yuzun 1512.

Lepiota oreadiformis Velen.: Locality 19, near road, 03.10.2011, Akata \& Yuzun 972.

Leucoagaricus barssii (Zeller) Vellinga: Locality 34, near road, 17.10. 2012, Akata \& Yuzun 1434.

Lycoperdon echinatum Pers.: Locality 31, spruce and beech mixed forest, 20.10.2011, Akata \& Yuzun 1033.

Lycoperdon excipuliforme (Scop.) Pers.: Locality 20, near spruce forest, 03.10.2011, Akata \& Yuzun 980.

Lycoperdon lividum Pers.: Locality 32, near road, 20.10.2011, Akata \& Yuzun 1036.

Lycoperdon molle Pers.: Locality 14, spruce and beech mixed forest, 01.10.2011, Akata \& Yuzun 955.

Lycoperdon perlatum Pers.: Locality 12, spruce forest, 25.09.2011, Akata \& Yuzun 950.

Lycoperdon pratense Pers.: Locality 21, near road, 10.10.2011, Akata \& Yuzun 991.

Lycoperdon pyriforme Schaeff.: Locality 11, on spruce stump, 25.09.2011, Akata \& Yuzun 945.

Lycoperdon utriforme Bull:: Locality 6, pastureland, 10.09.2011, Akata \& Yuzun 908.

Macrolepiota konradii (Huijsman ex P.D. Orton) M.M. Moser: Locality 37, near spruce forest, 26.10.2012, Akata \& Yuzun 1469.

Macrolepiota procera (Scop.) Singer: Locality 8, spruce and beech mixed forest, 11.09.2011, Akata \& Yuzun 922.

\section{Family Amanitaceae}

Amanita caesarea (Scop.) Pers.: Locality 26, spruce and beech mixed forest, 08.09.2012, Akata \& Yuzun 1113.

Amanita citrina (Pers.) Pers.: Locality 3, spruce and beech mixed forest, 29.08.2011, Akata \& Yuzun 878.

Amanita gemmata (Fr.) Bertill.: Locality 29, spruce and beech mixed forest, 19.10.2011, Akata \& Yuzun 1029.

Amanita muscaria (L.) Lam.: Locality 32, near spruce and beech mixed forest, 20.10.2011, Akata \& Yuzun 1040. 
Amanita pantherina (DC.) Krombh.: Locality 9, spruce and beech mixed forest, 15.09.2011, Akata \& Yuzun 939.

Amanita phalloides (Vaill. ex Fr.) Link: Locality 26, spruce and beech mixed forest, 08.09.2012, Akata \& Yuzun 1120.

Amanita rubescens Pers.: Locality 16, spruce and beech mixed forest, 02.10.2011, Akata \& Yuzun 966.

Amanita vaginata (Bull.) Lam.: Locality 25, spruce and beech mixed forest, 14.10.2011, Akata \& Yuzun 1025.

\section{Family Auriculariaceae}

Auricularia mesenterica (Dicks.) Pers.: Locality 25, on alder stump, 14.10.2011, Akata \& Yuzun 1022.

Exidia nigricans (With.) P. Roberts: Locality 35, on beech branch, 17.10.2012, Akata \& Yuzun 1460.

Pseudohydnum gelatinosum (Scop.: Fr.) Karst.: Locality 22, on spruce stump, 12.10.2011, Akata \& Yuzun 994.

\section{Family Auriscalpiaceae}

Lentinellus cochleatus (Pers.) P. Karst: Locality 23, on spruce stump, 12.10.2011, Akata \& Yuzun 1005.

\section{Family Bankeraceae}

Hydnellum auratile (Britzelm.) Maas Geest.: Locality 10, spruce forest, 25.09.2011, Akata \& Yuzun 946.

Hydnellum spongiosipes (Peck) Pouzar: Locality 36, spruce and beech mixed forest, 26.10.2012, Akata \& Yuzun 1461.

Sarcodon imbricatus (L.) P. Karst.: Locality 15, spruce forest, 02.10.2011, Akata \& Yuzun 965.

\section{Family Bolbitiaceae}

Conocybe arrhenii (Fr.) Kits van Wav.: Locality 21, near road, 10.10.2011, Akata \& Yuzun 986.

Conocybe tenera (Schaeff.) Fayod: Locality 6, pastureland, 10.09.2011, Akata \& Yuzun 904.

\section{Family Boletaceae}

Boletus chrysenteron Bull.: Locality 28, spruce and beech mixed forest, 19.09.2012, Akata \& Yuzun 1160.

Boletus edulis Bull.: Locality 23, spruce forest, 12.10.2011, Akata \& Yuzun 1004.

Boletus erythropus Pers.: Locality 17, spruce forest, 06.09.2011, Akata \& Yuzun 929.

\section{Family Bondarzewiaceae}

Heterobasidion annosum (Fr.) Bref.: Locality 22, on spruce stump, 13.06.2013, Akata \& Yuzun, 1685

\section{Family Cantharallaceae}

Cantharellus cibarius Fr.: Locality 18, spruce and beech mixed forest, 11.09.2011, Akata \& Yuzun 926.
Craterellus cornucopioides (L.) Pers.: Locality 40, spruce and beech mixed forest, 27.10.2012, Akata \& Yuzun 1541.

Craterellus tubaeformis (Fr.) Quél.: Locality 9, spruce and beech mixed forest, 15.09.2011, Akata \& Yuzun 942.

\section{Family Clavariadelphaceae}

Clavariadelphus truncatus (Quél.) Donk: Locality 18, spruce and beech mixed forest, 11.09.2011, Akata \& Yuzun 925.

\section{Family Clavulinaceae}

Clavulina cinerea (Bull.) J. Schröt.: Locality 40, spruce and beech mixed forest, 27.10.2012, Akata \& Yuzun 1549.

Clavulina rugosa (Bull.) J. Schröt.: Locality 14, spruce and beech mixed forest, 01.10.2011, Akata \& Yuzun 962.

\section{Family Dacrymycetaceae}

Calocera cornea (Batsch) Fr.: Locality 23, on spruce stump, 12.10.2011, Akata \& Yuzun 993.

Calocera viscosa (Pers.) Fr.: Locality 15, on spruce stump, 02.10.2011, Akata \& Yuzun 967.

Dacrymyces stillatus Nees: Locality 40, on beech stump, 27.10.2012, Akata \& Yuzun 1527.

\section{Family Diplocystidiaceae}

Astraeus hygrometricus (Pers.) Morgan: Locality 19, near road, 03.10.2011, Akata \& Yuzun 983.

\section{Family Entolomataceae}

Entoloma asprellum (Fr.) Fayod: (Kaya et al., 2013).

Entoloma rhodopolium (Fr.) P. Kumm.: Locality 25, spruce and beech mixed forest, 04.10.2012, Akata \& Yuzun 1254.

\section{Family Fistulinaceae}

Fistulina hepatica (Schaeff.) With.: Locality 40, on common hazel stump, 27.10.2012, Akata \& Yuzun 1530.

\section{Family Fomitopsidaceae}

Daedalea quercina (L.) Pers.: Locality 1, on chesnut stump, 23.08.2011, Akata \& Yuzun 872.

Fomitopsis pinicola (Sw.) P. Karst.: Locality 32, on spruce stump, 20.10.2011, Akata \& Yuzun 1038.

Laetiporus sulphureus (Bull.) Murrill: Locality 2, on poplar, 23.08.2011, Akata \& Yuzun 868.

\section{Family Ganodermataceae}

Ganoderma lucidum (Curtis) P. Karst.: Locality 18, on beech root, 11.09.2011, Akata\&Yuzun 930.

\section{Family Geastraceae}

Geastrum fimbriatum Fr.: Locality 25, spruce and beech mixed forest, 19.10.2011, Akata \& Yuzun 1032 
Geastrum quadrifidum DC. ex Pers.: Locality 37, spruce forest, 26.10.2012, Akata \& Yuzun 1480.

Geastrum rufescens Pers.: Locality 9, spruce and beech mixed forest, 15.09.2011, Akata \& Yuzun 940.

Geastrum triplex Jungh: Locality 27, spruce and beech mixed forest, 08.09.2012, Akata \& Yuzun 1154.

\section{Family Gomphaceae}

Gomphus clavatus (Pers.) Gray: Locality 40, spruce and beech mixed forest, 27.10.2012, Akata \& Yuzun 1603.

Phaeoclavulina flaccida (Fr.) Giachini: Locality 31, spruce and beech mixed forest, 04.10.2012, Akata \& Yuzun, 1294

Ramaria flavescens (Schaeff.) R.H. Petersen: Locality 40, spruce and beech mixed forest, 27.10.2012, Akata \& Yuzun 1533.

\section{Family Hydnaceae}

Hydnum repandum L.: Locality 4, spruce and beech mixed forest, 06.09.2011, Akata \& Yuzun 887.

\section{Family Hydnangiaceae}

Laccaria laccata (Scop.) Cooke: Locality 26, spruce and beech mixed forest, 08.09.2012, Akata \& Yuzun 1118.

\section{Family Hygrophoraceae}

Cuphophyllus virgineus (Wulfen) Kovalenko: Locality 40, spruce and beech mixed forest, 27.10.2012, Akata \& Yuzun 1531.

Gliophorus psittacinus (Schaeff.) Herink: Locality 25, spruce and beech mixed forest, 04.10.2012, Akata \& Yuzun 1298.

Hygrocybe acutoconica (Clem.) Singer: Locality 28, spruce and beech mixed forest, 11.10.2012, Akata \& Yuzun 1326.

Hygrocybe conica (Schaeff.) P. Kumm.: Locality 6, pastureland, 10.09.2011, Akata \& Yuzun 915.

Hygrophorus agathosmus (Fr.) Fr.: Locality 30, spruce and beech mixed forest, 04.10.2012, Akata \& Yuzun 1265.

Hygrophorus chrysodon (Batsch) Fr.: Locality 40, spruce and beech mixed forest, 27.10.2012, Akata \& Yuzun 1537.

Hygrophorus erubescens (Fr.) Fr.: Locality 31, spruce and beech mixed forest, 29.09.2012, Akata \& Yuzun 1229.

Hygrophorus pudorinus (Fr.) Fr.: Locality 18, spruce and beech mixed forest, 11.09.2011, Akata \& Yuzun 932.

\section{Family Hymenochaetaceae}

Hymenochaete rubiginosa (Dicks.) Lév.: Locality 31, on stump, 29.09.2012, Akata \& Yuzun 1225.
Phellinus viticola (Schwein.) Donk: Locality 14, on spruce stump, 11.10.2012, Akata \& Yuzun 1372.

\section{Family Hymenogastraceae}

Hebeloma sinapizans (Paulet) Gillet: Locality 40, spruce and beech mixed forest, 27.10.2012, Akata \& Yuzun 1525.

\section{Incertae sedis}

Panaeolus papilionaceus (Bull.) Quél.: Locality 32, near road, on cow dung, 20.10.2011, Akata \& Yuzun 1034.

\section{Family Inocybaceae}

Crepidotus caspari Velen.: Locality 30, on beech branch, 04.10.2012, Akata \& Yuzun 1261.

Crepidotus epibryus (Fr.) Quél.: Locality 25, on beech leaves, 04.10.2012, Akata \& Yuzun 1291.

Inocybe asterospora Quél.: Locality 9, spruce and beech mixed forest, 15.09.2011, Akata \& Yuzun 937.

Inocybe cervicolor (Pers.) Quél.: Locality 31, spruce and beech mixed forest, 11.10.2012, Akata \& Yuzun 1376.

Inocybe geophylla (Fr.) P. Kumm.: Locality 40, spruce and beech mixed forest, 27.10.2012, Akata \& Yuzun 1555 .

Inocybe praetervisa Quél.: Locality 4, spruce and beech mixed forest, 06.09.2011, Akata \& Yuzun 890.

Inocybe rimosa (Bull.) P. Kumm.: Locality 22, spruce forest,12.10.2011, Akata \& Yuzun 996.

\section{Family Lyophyllaceae}

Asterophora lycoperdoides (Bull.) Ditmar Locality 25, on rotten Russula Pers., 04.10.2012, Akata \& Yuzun 1287.

Asterophora parasitica (Bull. ex Pers.) Singer: Locality 28, on rotten Russula, 11.10.2012, Akata \& Yuzun 1311.

\section{Family Marasmiaceae}

Macrocystidia cucumis (Pers.) Joss.: Locality 31, spruce and beech mixed forest, 29.09.2012, Akata \& Yuzun 1240.

Marasmius bulliardii Quél.: Locality 30, on beech leaves, 04.10.2012, Akata \& Yuzun 1272.

Marasmius oreades (Bolton) Fr.: Locality 14, spruce and beech mixed forest, 11.10.2012, Akata \& Yuzun 1373.

Marasmius rotula (Scop.) Fr.: Locality 25, on beech branch, 04.10.2012, Akata \& Yuzun 1259.

\section{Family Mycenaceae}

Mycena epipterygia (Scop.) Gray: Locality 40, on the remains of spruce wood, 27.10.2012, Akata \& Yuzun 1563. 
Mycena galopus (Pers.) P. Kumm.: Locality 31, the remains of beech wood, 29.09.2012, Akata \& Yuzun 1236.

Mycena pura (Pers.) P. Kumm.: Locality 40, spruce and beech mixed forest, 27.10.2012, Akata \& Yuzun 1539 .

Mycena sanguinolenta (Alb. \& Schwein.) P. Kumm.: Locality 30, spruce and beech mixed forest, 04.10.2012, Akata \& Yuzun 1256.

Mycena silvae-nigrae Maas Geest. \& Schwöbel: Locality 16, on spruce stump, 13.10.2011, Akata \& Yuzun 1009.

Mycena stipata Maas Geest. \& Schwöbel: Locality 28, on spruce stump, 11.10.2012, Akata \& Yuzun 1330.

Panellus stipticus (Bull.) P. Karst.: Locality 14, on beech branch, 11.10.2012, Akata \& Yuzun 1348.

\section{Meruliaceae}

Bjerkandera adusta (Willd.) P. Karst.: Locality 22, on beech stump, 13.06.2013, Akata \& Yuzun 1689.

\section{Family Omphalotaceae}

Gymnopus confluens (Pers.) Antonín, Halling \& Noordel.: Locality 14, spruce and beech mixed forest, 11.10.2012, Akata \& Yuzun 1362.

Gymnopus dryophilus (Bull.) Murrill: Locality 25, 04.10.2012, spruce and beech mixed forest, Akata \& Yuzun 1281.

Gymnopus erythropus (Pers.) Antonín, Halling \& Noordel.: Locality 31, on beech stump, 29.09.2012, Akata \& Yuzun 1210.

Mycetinis scorodonius (Fr.) A.W. Wilson \& Desjardin: Locality 28, spruce and beech mixed forest, 11.10.2012, Akata \& Yuzun 1332.

Rhodocollybia butyracea (Bull.) Lennox: Locality 30, spruce and beech mixed forest, 04.10.2012, Akata \& Yuzun 1299.

\section{Family Paxillaceae}

Paxillus involutus (Batsch) Fr.: Locality 16, spruce forest, 13.10.2011, Akata \& Yuzun 1008.

\section{Family Phallaceae}

Clathrus ruber P. Micheli ex Pers.: Locality 18, spruce and beech mixed forest, 11.09.2011, Akata \& Yuzun 921.

Mutinus caninus (Huds.) Fr.: Locality 25, spruce and beech mixed forest, 04.10.2012, Akata \& Yuzun 1273.

Phallus impudicus L.: Locality 9, 15.09.2011, spruce and beech mixed forest, Akata \& Yuzun 938.

\section{Family Physalacriaceae}

Armillaria mellea (Vahl) P. Kumm.: Locality 25, on beech stump, 04.10.2012, Akata \& Yuzun 1277.
Mucidula mucida (Schrad.) Pat.: Locality 40, on beech stump, 27.10.2012, Akata \& Yuzun 1567.

Hymenopellis radicata (Relhan) R.H. Petersen: Locality 30, on beech root, 04.10.2012, Akata \& Yuzun 1290.

Xerula pudens (Pers.) Singer: Locality 40, spruce and beech mixed forest, 27.10.2012, Akata \& Yuzun 1551.

\section{Family Pleurotaceae}

Pleurotus ostreatus (Jacq.) P. Kumm.: Locality 30, on beech stump, 04.10.2012, Akata \& Yuzun 1276.

\section{Family Pluteaceae}

Pluteus atromarginatus (Konrad) Kühner: Locality 9, on spruce stump, 15.09.2011, Akata \& Yuzun 941.

\section{Family Polyporaceae}

Cerioporus squamosus (Huds.) Quél.: Locality 1, on stump, 23.08.2011, Akata \& Yuzun 877.

Lenzites betulina (L.) Fr.: Locality 31, on beech branch, 29.09.2012, Akata \& Yuzun 1233.

Panus conchatus (Bull.) Fr. Locality 28, on beech stump, 11.10.2012, Akata \& Yuzun 1312.

Polyporus melanopus (Pers.) Fr.: Locality 30, on beech stump, 04.10.2012, Akata \& Yuzun 1257.

Picipes badius (Pers.) Zmitr. \& Kovalenko: Locality 5, on alder stump, 10.09.2011, Akata \& Yuzun 900.

Trametes hirsuta (Wulfen) Lloyd: Locality 40, on beech stump, 27.10.2012, Akata \& Yuzun 1526.

Trametes versicolor (L.) Lloyd: Locality 31, on beech stump, 29.09.2012, Akata \& Yuzun 1246.

\section{Family Psathyrellaceae}

Coprinellus disseminatus (Pers.) J.E. Lange: Locality 40, on beech stump, 27.10.2012, Akata \& Yuzun 1570.

Coprinellus micaceus (Bull.) Vilgalys, Hopple \& Jacq. Johnson: Locality 14, on the remains of beech wood, 01.10.2011, Akata \& Yuzun 964.

Coprinellus xanthothrix (Romagn.) Vilgalys, Hopple \& Jacq. Johnson: Locality 40, on the remains of beech wood, 27.10.2012, Akata \& Yuzun 1545.

Coprinopsis atramentaria (Bull.) Redhead, Vilgalys \& Moncalvo: Locality 6, pastureland, 10.09.2011, Akata \& Yuzun 906.

\section{Family Russulaceae}

Lactarius aurantiacus (Pers.) Gray: Locality 25, spruce and beech mixed forest, 14.10.2011, Akata \& Yuzun 1026.

Lactarius piperatus (L.) Pers.: Locality 29, spruce and beech mixed forest, 13.10.2011, Akata \& Yuzun 1006.

Lactarius scrobiculatus (Scop.) Fr.: Locality 14, spruce and beech mixed forest, 11.10.2012, Akata \& Yuzun 1343. 
Lactarius vellereus (Fr.) Fr.: Locality 40, spruce and beech mixed forest, 27.10.2012, Akata \& Yuzun 1561.

Lactarius volemus (Fr.) Fr.: Locality 29, spruce and beech mixed forest, 13.10.2011, Akata \& Yuzun 1002.

Russula chloroides (Krombh.) Bres.: Locality 31, spruce and beech mixed forest, 29.09.2012, Akata \& Yuzun 1217.

Russula cyanoxantha (Schaeff.) Fr.: Locality 9, spruce and beech mixed forest, 15.09.2011, Akata \& Yuzun 941.

Russula emetica (Schaeff.) Pers.: Locality 25, spruce and beech mixed forest, 04.10.2012, Akata \& Yuzun 1266.

\section{Family Schizophyllaceae}

Schizophyllum commune Fr.: Locality 2, on fallen branch, 23.08.2011, Akata \& Yuzun 873.

\section{Family Sclerodermataceae}

Scleroderma bovista Fr.: Locality 32, near road, 20.10.2011, Akata \& Yuzun 1039.

Scleroderma polyrhizum (J.F. Gmel.) Pers.: Locality 40, spruce and beech mixed forest, 27.10.2012, Akata \& Yuzun 1522.

\section{Family Stereaceae}

Stereum hirsutum (Willd.) Pers.: Locality 22, on beech stump, 13.06.2013, Akata \& Yuzun 1687.

Stereum rameale (Schwein.) Burt: Locality 36, on beech stump, 26.10.2012, Akata \& Yuzun 1486.

\section{Family Strophariaceae}

Hypholoma fasciculare (Huds.) P. Kumm.: Locality 40, on common hazel stump, 27.10.2012, Akata \& Yuzun 1521.

Pholiota lucifera (Lasch) Quél.: Locality 25, on alder stump, 14.10.2011, Akata \& Yuzun 1028.

Stropharia aeruginosa (Curtis) Quél.: Locality 40, spruce and beech mixed forest, 27.10.2012, Akata \& Yuzun 1553.

Stropharia rugosoannulata Farl. ex Murrill: Locality 39, near spruce and beech mixed forest, 27.10.2012, Akata \& Yuzun 1509.

\section{Family Suillaceae}

Suillus collinitus (Fr.) Kuntze: Locality 1, under pine, 13.10.2011, Akata \& Yuzun 1016.

Suillus granulatus (L.) Roussel: Locality 38, spruce and beech mixed forest, 26.10.2012, Akata \& Yuzun 1486.

\section{Family Tapinellaceae}

Tapinella atrotomentosa (Batsch) Šutara: Locality 13, on spruce stump, 01.10.2011, Akata \& Yuzun 957.

\section{Family Tricholomataceae}

Clitocybe foetens Melot: Locality 5, spruce and beech mixed forest, 06.09.2011, Akata \& Yuzun 893.
Clitocybe gibba (Pers.) P. Kumm.: Locality 31, spruce and beech mixed forest, 11.10.2012, Akata \& Yuzun 1368.

Clitocybe nebularis (Batsch) P. Kumm. Locality 40, spruce and beech mixed forest, 27.10.2012, Akata \& Yuzun 1556.

Clitocybe odora (Bull.) P. Kumm.: Locality 27, spruce and beech mixed forest, 08.09.2012, Akata \& Yuzun 1151 .

Delicatula integrella (Pers.) Fayod: Locality 40, on common hazel stump, 27.10.2012, Akata \& Yuzun 1550.

Lepista nuda (Bull.) Cooke: Locality 8, spruce and beech mixed forest, 11.09.2011, Akata \& Yuzun 917.

Myxomphalia maura (Fr.: Fr.) Hora: Locality 37, spruce forest, 26.10.2012, Akata \& Yuzun 1468.

Paralepista flaccida (Sowerby) Vizzini: Locality 25, spruce and beech mixed forest, 14.10.2011, Akata \& Yuzun 1024.

Tricholoma terreum (Schaeff.) P. Kumm.: Locality 16, spruce forest, 02.10.2011, Akata \& Yuzun 970.

Tricholoma vaccinum (Schaeff.) P. Kumm.: Locality 22, spruce forest, 12.10.2011, Akata \& Yuzun 995.

Tricholomopsis rutilans (Schaeff.) Singer: Locality 17, on spruce stump, 06.09.2011, Akata \& Yuzun 892.

\section{Family Tremellaceae}

Tremella encephala Pers.: Locality 38, on spruce stump, 26.10.2012, Akata \& Yuzun 1497.

Tremella foliacea Pers.: Locality 14, on beech stump, 01.10.2011, Akata \& Yuzun 954.

Tremella mesenterica Retz.: Locality 27, on beech stump, 08.09.2012, Akata \& Yuzun 1186.

\section{Discussion}

Uzungöl Nature Park was a region where data on its macrufungal diversity was lacking until the present study. Previous studies in the region revealed presence of only 7 macrofungi species (Akata 2012a,b, Akata \& Kaya 2013a,b, Kaya et al. 2013). Our present detailed sampling in the region added 205 species to the macromycota of the region, indicating a relatively rich biodiversity for a relatively small area. We, therefore included all recorded species in our present list with which we listed 212 species belonging to 129 genera and 64 families inside the boundaries of Uzungöl Nature Park. The list included 46 members in Ascomycota (Caloscyphaceae, Chlorociboriaceae, Diatrypaceae, Geoglossaceae, Hyaloscyphaceae, Hypocreaceae, Lachnaceae, Leotiaceae, Morchellaceae, Rutstroemiaceae, Sarcoscyphaceae and Sarcosomataceae and Incertae sedis, 1; Cudoniaceae and Pezizaceae 3; Helvellaceae 4; Helotiaceae 5; Xylariaceae 8 and Pyronemataceae 10) and 166 members in Basidiomycota (Auriscalpiaceae, Bondarzewiaceae, Clavariadelphaceae, Diplocystidiaceae, 
Fistulinaceae, Ganodermataceae, Hydnaceae, Hydnangiaceae, Hymenogastraceae, Meruliaceae, Paxillaceae, Pleurotaceae, Pluteaceae, Schizophyllaceae and Tapinellaceae and Incertae sedis 1; Bolbitiaceae, Clavulinaceae, Entolomataceae, Hymenochaetaceae, Lyophyllaceae, Sclerodermataceae, Stereaceae and Suillaceae 2; Auriculariaceae, Bankeraceae, Boletaceae, Cantharallaceae, Dacrymycetaceae, Fomitopsidaceae, Gomphaceae, Phallaceae and Tremellaceae 3; Geastraceae, Marasmiaceae, Physalacriaceae, Strophariaceae and Psathyrellaceae 4; Omphalotaceae 5; Inocybaceae, Mycenaceae and Polyporaceae 7; Amanitaceae, Hygrophoraceae and Russulaceae 8; Tricholomataceae 11 and Agaricaceae 26).

The most widespread macrofungi species in the nature park were; Armillaria mellea, Boletus chrysenteron, Cantharellus cibarius, Cyathus striatus, Coprinellus disseminatus, Fomitopsis pinicola, Hydnum repandum, Hymenopellis radicata, Hypholoma fasciculare, Inocybe geophylla, Laccaria laccata, Lactarius volemus, Lycoperdon lividum, L. perlatum, Macrolepiota procera,

\section{References}

1. Akata, I. 2012a. A New Ascomycete Genus Record for Turkey. Ot Sistematik Botanik Dergisi, 19 (1): 113-116.

2. Akata, I. 2012b. A new Ascomycete family record for Turkish Macromycota. Biological Diversity and Conservation, 5(3): 37-39.

3. Akata, I. \& Doğan, H.H. 2015. Orbiliaceae for Turkish Ascomycota: Three new records. Bangladesh Journal of Botany, 44(1): 91-95.

4. Akata, I. \& Kaya, A. 2012. Two New Additions to Turkish Ascomycota. International Journal of Botany, 8(2): 79-81.

5. Akata, I. \& Kaya, A. 2013a. Two earth-tongue genera new for Turkey. Mycotaxon, 125: 87-90.

6. Akata, I. \& Kaya, A. 2013b. Three pyronemataceous macrofungi genera new to Turkish Mycota. Turkish Journal of Botany, 37(5): 977-980.

7. Akata, I., Kaya, A. \& Uzun, Y. 2011. New additions to Turkish Pyronemataceae. Biological Diversity and Conservation, 4(1): 182-185.

8. Akata, I., Kaya, A. \& Uzun, Y. 2012. New Ascomycete records for Turkish macromycota. Turkish Journal of Botany, 36(4): 420-424.

9. Akata, I., Kaya, A. \& Uzun, Y. 2014. Macromycetes determined in Yomra (Trabzon) district. Turkish Journal of Botany, 38(5): 999-1012.

10. Akata, I., Kaya, A. \& Uzun, Y. 2016a. Macrofungal diversity of Zigana Mountain (Gümüşhane/Turkey). Biological Diversity and Conservation, 9(2): 57-69.

11. Akata, I., Kaya, A. \& Uzun, Y. 2016b. Two New Genus Records for Turkish Helotiales. Kastamonu Üniversitesi Orman Fakültesi Dergisi, 16(1): 131-134.

12. Akata, I., Kaya, E., Yılmaz, İ., Bakırcı, S. \& Bayram, R. 2015. Türkiye'de Yetişen Alfa Amanitin İçeren Mantarlar. Düzce Tip Fakültesi Dergisi, 17(1): 39-44.
Mycena pura, Sarcodon imbricatus, Schizophyllum commune, Stereum hirsutum, Trametes versicolor, Tremella mesenterica and Xylaria hypoxylon.

Although 50 of 212 species determined in the study area are know to be suitable for human consumption only 5 species (Cantharellus cibarius, Hydnum repandum, Macrolepiota procera, Lactarius volemus and Sarcodon imbricatus) are commonly eaten by local people. There are also 17 poisonous species (Agaricus xanthodermus, Amanita gemmata, A. muscaria, A. pantherina, A. phalloides, Boletus erythropus, Coprinopsis atramentaria, Entoloma rhodopolium, Hypholoma fasciculare, Inocybe geophylla, I. rimosa, Lactarius piperatus, Lepiota clypeolaria, L. cristata, Paxillus involutus, Russula emetica and Tapinella atrotomentosa) determined in the study area among which A. phalloides is the most dangerous one that is responsible for $95 \%$ of deadly mushroom poisonings in Turkey (Akata et al. 2015). Due to collection and consumption of only well known edible mushrooms generally by local people, no poisoning case in the nature park has been reported so far.

13. Allı, H., Şen, İ. \& Altuntaş, D. 2016. Macrofungi of İznik Province. Communications. Faculty of Science University of Ankara, Series C: Biology, 25(1-2): 7-24.

14. Arıkan, H., Tosunoğlu, M., Atatür, M.K. \& Göçmen, B. 2007. Some Comments on the Breeding Biology of Pelodytes caucasicus Boulenger, 1896 (Anura: Pelodytidae) from Uzungöl, Northeast Anatolia. Turkish Journal of Zoology, 31(1): 53-64.

15. Atasoy, M. 2010. Monitoring land use changes in tourism centers with VGIS: Uzungöl case study. Scientific Research and Essays, 5(8): 790-798.

16. Bas, C., Kuyper, T.W., Noordeloos, M.E. \& Vellinga, E.C. 1988. Flora Agaricina Neerlandica-Critical monographs on the families of agarics and boleti occuring in the Netherlands. Volume 1. Entolomataceae. A.A. Balkema, Rotterdam, Netherlands, 182 pp.

17. Bas, C., Kuyper, T.W., Noordeloos, M.E. \& Vellinga, E.C. 1990. Flora Agaricina Neerlandica-Critical monographs on the families of agarics and boleti occuring in the Netherlands. Volume 2. Pluteaceae, Tricholomataceae. A. A. Balkema, Rotterdam, Netherlands, 137 pp.

18. Bas, C., Kuyper, T.W., Noordeloos, M.E. \& Vellinga, E.C. 1995. Flora Agaricina Neerlandica-Critical monographs on the families of agarics and boleti occuring in the Netherlands. Volume 3. Tricholomataceae. A. A. Balkema, Rotterdam, Netherlands, $183 \mathrm{pp}$.

19. Baydar, S. \& Sesli, E. 1994. The macromycetes determined in Akçaabat District of Trabzon Province. Turkish Journal of Botany, 18: 99-101.

20. Boertmann, D. 1996. The Genus Hygrocybe (Fungi of Northern Europe, Vol. 1). Danish Mycological Society, Copenhagen, Denmark, 184 pp.

21. Breitenbach, J. \& Kränzlin, F. 1984. Fungi of Switzerland, Vol: 1, Ascomycetes. Verlag Mykologia CH-6000 Luzern 9, Switzerland, $310 \mathrm{pp}$. 
22. Breitenbach, J. \& Kränzlin, F. 1986. Fungi of Switzerland. Vol: 2, Nongilled Fungi. Verlag Mykologia CH-6000 Luzern 9, Switzerland, 412 pp.

23. Breitenbach, J. \& Kränzlin, F. 1991. Fungi of Switzerland. Vol: 3, Boletes and Agarics 1. Part. Verlag Mykologia CH6000 Luzern 9, Switzerland, 361 pp.

24. Breitenbach, J. \& Kränzlin, F. 1995. Fungi of Switzerland. Vol: 4, Agarics 2. Part. Verlag Mykologia CH-6000 Luzern 9, Switzerland, $368 \mathrm{pp}$.

25. Breitenbach, J. \& Kränzlin, F. 2000. Fungi of Switzerland. Vol: 5, Agarics 3. Part. Verlag Mykologia CH-6000 Luzern 9, Switzerland, $338 \mathrm{pp}$.

26. Cannon, P.F. \& Kirk, P.M. 2007. Fungal families of the world. CAB International, Wallingford, $456 \mathrm{pp}$.

27. Demirel, K., Acar, İ. \& Ömeroğlu Boztepe, G. 2016. Lice (Diyarbakır) Yöresi Makrofungusları. Mantar Dergisi, 7(1): 29-39.

28. Doğan, H.H \& Öztürk, Ö. 2015. Six new Russula records from Turkey. Mycotaxon, 130(4): 1117-1124.

29. Doğan, H.H. \& Kurt, F. 2016. New macrofungi records from Turkey and macrofungal diversity of Pozant1-Adana. Turkish Journal of Botany, 40(2): 209-217.

30. Dülger, B. \& Akata, I. 2016. Lasiosphaeria ovina, A first record for family Lasiosphaeriaceae in Turkey. Mantar Dergisi, 7(1): 88-91.

31. Fan, L., Liu, B. \& Liu, H.Y. 1994. The Gasteromycetes of China. Gebrüder Borntraeger, Stuttgart, 72 pp.

32. Hansen, L. \& Knudsen, H. 1992. Nordic Macromycetes. Volume 2. Polyporales, Boletales, Agaricales, Russulales. Nordsvamp, Copenhagen, Denmark, 474 pp.

33. Hansen, L. \& Knudsen, H. 1997. Nordic Macromycetes. Volume 3. Heterobasidoid, Aphyllophoroid, and Gastromycetoid Basidiomycetes. Nordsvamp, Copenhagen, Denmark, 444 pp.

34. Hansen, L. \& Knudsen, H. 2000. Nordic Macromycetes. Volume 1. Ascomycetes. Nordsvamp, Copenhagen, Denmark, 209 pp.

35. Kaya, A., Akata, I. \& Uzun, Y. 2013. Two new records for Turkish Agaricales. Biological Diversity and Conservation, 6(3): 150-152.
36. Kirk, P.M., Cannon, P.F., Minter, D.W. \& Stalpers, J.A. 2008. Dictionary of the fungi, 10th ed. CAB International. Wallingford, UK, $771 \mathrm{pp}$.

37. Kränzlin, F. 2005. Fungi of Switzerland. Volume 6. Russulaceae 2. Verlag Mykologia, Switzerland, 319 pp.

38. Kuyper, T.W. 1986. A Revision of the Genus Inocybe in Europe. Rijksherbarium, Leiden, 247 pp.

39. Medardi, G. 2006. Atlante fotografico degli Ascomiceti d'Italia. A.M.B. Fondazione, Centro Studi Micologici, Italia, $454 \mathrm{pp}$

40. Pegler, D.N., Roberts, P.J. \& Spooner, B.M. 1997. British Chanterelles and Tooth Fungi. Royal Botanic Gardens, Kiew, 114 pp.

41. Ryvarden, L. \& Gilbertson, R.L. 1993. European Polypores Vol: 1-2, Synopsis Fungorum 6. Fungiflora, Oslo, Norway, $745 \mathrm{pp}$.

42. Sesli, E. 1993. Trabzon ili Maçka yöresi makrofungusları. Turkish Journal of Botany, 17: 179-182.

43. Sesli, E. \& Denchev, C.M. 2008. Checklists of the myxomycetes, larger ascomycetes, and larger basidiomycetes in Turkey. Mycotaxon, 106: 65-67.

44. Sesli, E., Türkekul, İ., Akata, I. \& Niskanen, T. 2016. New records of Basidiomycota from Trabzon, Tokat, and İstanbul provinces in Turkey. Turkish Journal of Botany, 40(5): 531-545.

45. Sunhede, S. 1989. Geastraceae (Basidiomycotina), Synopsis Fungorum 1. Fungiflora, Oslo, Norway, 533 pp.

46. Uzun, Y., Acar, İ, Akçay, M.E. \& Akata, I. 2014. Additions to the Turkish Discomycetes. Turkish Journal of Botany, 38(3): 617-622.

47. Uzun, Y., Kaya, A., Akata, I, Keleş, A. \& Yakar, S. 2015. Notes on Turkish Hypocrea. Biological Diversity and Conservation, 8(2): 117-121.

48. Verep, B., Şahin, C., Çiloğlu, E. \& İmamoğlu, H.O. 2002. Uzungöl'ün İklimi ve Çevresel Sorunları Üzerine Bir Çalıșma. Atatürk Üniversitesi Ziraat Fakültesi Dergisi, 33(4): 353-358. 
